\title{
A CHECKLIST OF THE FAMILY LAUXANIIDAE (DIPTERA, LAUXANIOIDEA) IN VIETNAM, MAINLY WITH THE SUBFAMILY LAUXANIINAE
}

\author{
Hyun Suk Lee ${ }^{a^{*}}$ \\ ${ }^{a}$ The Faculty of Biology, Dalat University, Lamdong, Vietnam \\ *Corresponding author: Email: exuknight@gmail.com
}

\author{
Article history \\ Received: September $05^{\text {th }}, 2017 \mid$ Received in revised form: October $22^{\text {nd }}, 2017$ \\ Accepted: October $24^{\text {th }}, 2017$
}

\begin{abstract}
I reviewed the lauxaniid literature as many as possible and listed any lauxaniid species with Vietnamese records. In this paper, I provided a taxonomic checklist of 41 nominal species under two subfamilies Homoneurinae and Lauxaniinae. In total, the number of lauxaniid species in Vietnam became 101 species belong to 21 genera. The Homoneurinae consists of 6 genera and 63 species. The Lauxaniinae includes 15 genera and 38 species. These two checklists will be the fundamental base for comprehensive taxonomic research of lauxaniid fauna in Vietnam.
\end{abstract}

Keywords: Diptera; Checklist; Lauxaniidae; Lauxaniinae; Vietnam.

Article identifier: http://tckh.dlu.edu.vn/index.php/tckhdhdl/article/view/346

Article type: (peer-reviewed) Full-length review article

Copyright (C) 2018 The author(s).

Licensing: This article is licensed under a CC BY-NC-ND 4.0 


\title{
DANH LỤC CÁC LOÀI THUỘC HỌ LAUXANIIDAE (DIPTERA, LAUXANIOIDEA) TẠI VIẸT NAM, CHỦ YẾU THUỘC PHÂN HỌ LAUXANIINAE
}

\author{
Hyun Suk Lee a* $^{*}$
}

${ }^{a}$ Khoa Sinh học, Trường Đại học Đà Lạt, Lâm Đồng, Việt Nam

"Tác giả liên hệ: Email: exuknight@ gmail.com

\section{Lịch sử bài báo}

Nhận ngày 05 tháng 09 năm 2017 | Chỉnh sửa ngày 22 tháng 10 năm 2017

Chấp nhận đăng ngày 24 tháng 10 năm 2017

\section{Tóm tắt}

Dựa trên các tài liệu, danh luc các loài thuộc ho Lauxaniidae ở Việt Nam đã được thống kê, bài báo này cung cấp danh lục bao gồm 41 loài đã được định danh thuộc hai phân họ là Homoneurinae và Lauxaniinae. Tổng công, số luọng loài thuộc ho Lauxaniidae ở Việt Nam gồm 101 loài thuộc 21 giống. Trong đó, phân ho Homoneurinae gồm 63 loài thuộc 6 giống và phân họ Lauxaniinae gồm 38 loài thuộc 15 giống. Hai danh lục này là co sở cho nhũng nghiên cứu sâu hơn về khu hệ họ Lauxaniidae ở Việt Nam.

Từ khóa: Danh lục; Diptera; Lauxaniidae; Lauxaniinae; Việt Nam.

Mã số định danh bài báo: http://tckh.dlu.edu.vn/index.php/tckhdhdl/article/view/346

Loại bài báo: Bài báo tổng quan có bình duyệt

Bản quyền @ 2018 (Các) Tác giả.

Cấp phép: Bài báo này được cấp phép theo CC BY-NC-ND 4.0 


\section{INTRODUCTION}

There is no intensive taxonomic research about lauxaniid fauna in Vietnam except for Sasakawa (2001). Lee (2016) listed the Homoneurinae species previously recorded in Vietnam. Total 60 species under Homoneurinae was listed in that checklist. In this checklist, the author listed 16 genera, 41 species under 2 subfamilies based on the lauxaniid literature with Vietnamese records. Lee (2016) also provided the Homoneurinae checklist except for the genus Noonamyia. This genus has an ambiguous position in the classification of Lauxaniidae. Some researchers ranked this genus in the subfamily Lauxaniinae, whereas others moved this genus to the subfamily Homoneurinae. A systematic position of this genus is not verified clearly. In this checklist, I followed the classification by Sasakawa (1995).

The Homoneurinae consists of six genera and 63 species. The Lauxaniinae includes 15 genera and 38 species. The number of lauxaniid species in Vietnam became 101 species belong to 21 genera under two subfamilies in total. This diversity is just a small part of entire lauxaniid diversity in Vietnam. I am sure about their high diversity based on collecting experience until now. I will discover lauxaniid diversity as many as possible in Vietnam. This checklist will be the fundamental base for comprehensive taxonomic research of lauxaniid fauna in Vietnam.

\section{RESULTS}

\subsection{Subfamily Homoneurinae - Tribe Noonamyiini Sasakawa, 1995 \\ Genus Noonamyia Stuckengerg, 1971}

TS: Noonamyia palawanensis Stuckenberg, 1971.

1. Noonamyia euphlebia Sasakawa (1990, p. 131). TL: Malaysia, Negeri Sembilan, Pasoh Forest Reserve.

Vietnamese records: Noonamyia euphlebia: Sasakawa (1995, p. 153, distribution, new to Laos, Thailand, Vietnam); Sasakawa (2003, p. 63, distribution); Shi and Yang (2009, p. 40, distribution).

Distribution: Laos, Malaysia, Thailand, Vietnam.

2. Noonamyia flavoscutellata Shi, Yang, and Gaimari (2011, p. 684). TL: China, Guangxi Province, Jinxiu, Dayao Mountain, Yinshan, 1000m.

Vietnamese records: Noonamyia flavoscutellata; Shi et al. (2011, p. 684, description, diagnosis, distribution, key to species, male genitalic figure, remarks, wing photo).

Distribution: China (Guangxi), Thailand, Vietnam. 
3. Noonamyia sasakawai Papp (2006, p. 185). TL: Thailand, Doi Pui.

Vietnamese records: Noonamyia sasakawai; Shi et al. (2011, p. 687, description, diagnosis, distribution, habitus photo, key to species, male genitalic figure, new to Vietnam, remarks); Lee et al. (2016, p. 44, description, distribution, habitus photo).

Distribution: Thailand, Vietnam.

\subsection{Subfamily Lauxaniinae}

\section{Genus Cerataulina Hendel, 1907}

TS: Cerataulina longicornis Hendel, 1907.

4. Cerataulina nigrifrons Malloch (1927a, p. 102). TL: Indonesia, Sumatra, Bukittinggi.

Vietnamese records: Cerataulina nigrifrons; Sasakawa (2001, p. 41, diagnosis, distribution, male genitalic figure, new to Vietnam).

Distribution: Indonesia, Vietnam.

\section{Genus Chaetolauxania Kertész, 1915}

TS: Chaetolauxania sternopleuralis Kertész, 1915.

5. Chaetolauxania platystylis Sasakawa (2001, p. 42). TL: Vietnam, Laocai province, Sapa.

Vietnamese records: Chaetolauxania platystylis; Sasakawa (2001, p. 42, discussion, distribution, male genitalic figure).

Distribution: Vietnam.

Genus Melanopachycerina Malloch, 1927

TS: Pachycerina leucochaeta Meijere, 1914.

6. Melanopachycerina leucochaeta (Meijere, 1914, p. 236 (Pachycerina)). TL: Indonesia, Java, Nongkodjadjar.

Vietnamese records: Melanopachycerina leucochaeta; Sasakawa (2001, p. 40, diagnosis, distribution, new to Vietnam).

Distribution: Indonesia, Vietnam. 


\section{Genus Melinomyia Kertész, 1915}

TS: Melinomyia flava Kertész, 1915.

7. Melinomyia flava Kertész (1915, p. 500). TL: Taiwan, Kosempo.

Vietnamese records: Melinomyia flava; Sasakawa (2001, p. 43, distribution, new to Vietnam).

Distribution: China (Taiwan), Japan, South Korea, Vietnam.

\section{Genus Minettia Robineau-Desvoidy, 1830}

TS: Minettia nemorosa Robineau-Desvoidy, 1830 [= fasciata (Fallén, 1826)].

8. Minettia (Frendelia) fuscofasciata (Meijere, 1910, p. 125 (Lauxania)). TL: Indonesia, Java, Gunung Salak, near Bogor.

Vietnamese records: Minettia (Frendelia) fuscofasciata; Sasakawa (2001, p. 43, diagnosis, distribution, in Vietnamese key, male genitalic figure, new to Vietnam).

Distribution: China (Taiwan), Indonesia, Vietnam.

9. Minettia (Frendelia) quadrispinosa Malloch (1927b, p. 166). TL: Taiwan, Tsui Kutsu.

Vietnamese records: Minettia (Frendelia) quadrispinosa; Sasakawa (2001, p. 43, diagnosis, distribution, in Vietnamese key, new to Vietnam); Lee et al. (2016, p. 42, description, distribution, habitus photo).

Distribution: China (Taiwan), Vietnam.

10. Minettia (Frendelia) rufiventris (Macquart, 1848, p. 228 (Lauxania)). TL: Indonesia, Java.

Vietnamese records: Minettia (Frendelia) rufiventris; Sasakawa (2001, p. 43, diagnosis, distribution, in Vietnamese key, male genitalic figure, new to Vietnam); Sasakawa (2003, p. 61, distribution, new to Laos).

Distribution: China (Taiwan), India, Indonesia, Laos, Philippines, Vietnam.

11. Minettia (Minettia) imparispinosa Sasakawa (2001, p. 45). TL: Vietnam, Lamdong province, Fyan.

Vietnamese records: Minettia (Minettia) imparispinosa; Sasakawa (2001, p. 43, diagnosis, distribution, in Vietnamese key, male genitalic figure).

Distribution: Vietnam. 
12. Minettia (Minettiella) atrata (Meijere, 1910, p. 127 (Lauxania)).TL: Indonesia, Java, Pangerango.

Vietnamese records: Minettiella atrata; Shatalkin (1996, p. 147, key to species, distribution).

Distribution: Indonesia (Java), Malaysia, Vietnam.

13. Minettia (Minettiella) atratula (Meijere, 1924a, p. 49 (Lauxania)). TL: Indonesia, Sumatra, Gunung Talaman.

Vietnamese records: Minettia (Minettiella) atratula; Shi and Yang (2014, p. 83, diagnosis, distribution, redescription, male and female genitalic figure); Minettiella atratula; Shatalkin (1996, p. 147, key to species, distribution).

Distribution: China (Hainan, Taiwan), Indonesia (Sumatra), Vietnam.

14. Minettia (Minettiella) sasakawai Shi, Wang, and Yang (2011, p. 81). TL: Vietnam, Lamdong province, Fyan.

Vietnamese records: Sapromyza (Sapromyza) acrostichalis; Sasakawa (2001, p. 46, discussion, distribution, male genitalic figure); Sasakawa (2008, p. 44, in Oriental key, distribution); Minettia (Minettiella) sasakawai; Shi et al. (2011, p. 81, nom. nov. remarks, distribution, wing photo, male and female genitalic figure); Shi and Yang (2014, p. 92, diagnosis, distribution, redescription, remarks); Lee et al. (2016, p. 43, description, distribution, habitus photo).

Distribution: China (Hainan), Vietnam.

\section{Genus Pachycerina Macquart, 1835}

TS: Lauxania seticornis Fallén, 1820.

15. Pachycerina decemlineata Meijere (1914, p. 236). TL: Indonesia, Java, Nongkodjadjar.

Vietnamese records: Pachycerina decemlineata; Sasakawa (2001, p. 40, diagnosis, distribution, new to Vietnam); Sasakawa (2003, p. 58, distribution, new to Laos).

Distribution: China (Taiwan), Indonesia, Laos, Nepal, Vietnam.

16. Pachycerina flaviventris Malloch (1929, p. 20). TL: Philippines, Luzon, Laguna, Mt. Makiling. 
Vietnamese records: Pachycerina flaviventris; Sasakawa (2001, p. 40, diagnosis, distribution, male genitalic figure, new to Vietnam); Shi, Wu and Yang (2009, p. 504, description, diagnosis, distribution, habitus photo, in Chinese key, male genitalic figure); Lee et al. (2016, p. 46, description, distribution, habitus photo).

Distribution: China (Yunnan, Sichuan, Guizhou, Guangdong), Philippines (Luzon), Vietnam.

17. Pachycerina javana (Macquart, 1851, p. 247 (Sapromyza)). TL: Indonesia, Java.

Vietnamese records: Pachycerina javana; Lee et al. (2016, p. 45, description, distribution, habitus photo, new to Vietnam).

Distribution: China (Iiubei, Iiunan, Yunnan, Sichuan, Guizhou, Hainan, Taiwan), India (Assam), Japan (Ryukyus Islands), Indonesia (Java), Nepal, Philippines (Luzon), Malaysia (Sunda Islands), Sri Lanka, Vietnam.

\section{Genus Parapachycerina Stuckenberg, 1971}

TS: Parapachycerina munroi Stuckenberg, 1971.

18. Parapachycerina cuneifera (Kertész, 1913, p. 96 (Lauxania)). TL: Taiwan, Kosempo.

Vietnamese records: Parapachycerina cuneifera; Sasakawa (2001, p. 41, diagnosis, distribution, male genitalic figure, new to Vietnam).

Distribution: China (Taiwan), Vietnam.

\section{Genus Sapromyza Fallén, 1810}

TS: Musca flava Linnaeus, 1758 (a misidentification of Sapromyza obsoleta Fallén, 1820).

19. Sapromyza (Notiosapromyza) longimentula Sasakawa (2001, p. 47). TL: Vietnam, Lamdong province, Fyan.

Vietnamese records: Sapromyza (Notiosapromyza) longimentula; Sasakawa (2001, p. 46, diagnosis, distribution, in Vietnamese key, male genitalic figure); Sasakawa (2008, p. 43, in Oriental key, distribution); Shi, Li and Yang (2012, p. 186, diagnosis, habitus photo, remarks, distribution, new to China).

Distribution: China (Hainan), Vietnam.

20. Sapromyza (Notiosapromyza) pellopleura Sasakawa (2001, p. 48). TL: Vietnam, Lamdong province, Dalat, Mt. Lang Biang. 
Vietnamese records: Sapromyza (Notiosapromyza) pellopleura; Sasakawa (2001, p. 46, discussion, distribution, in Vietnamese key); Sasakawa (2008, p. 43, in Oriental key, distribution).

Distribution: Vietnam.

21. Sapromyza (Notiosapromyza) quadridentata Sasakawa (2001, p. 49). TL: Vietnam, Lamdong province, Dalat, Mt. Lang Biang.

Vietnamese records: Sapromyza (Notiosapromyza) quadridentata; Sasakawa (2001, p. 46, diagnosis, distribution, in Vietnamese key, male genitalic figure); Sasakawa (2008, p. 43, in Oriental key, distribution).

Distribution: Vietnam.

22. Sapromyza (Sapromyza) ctenophora Sasakawa (2001, p. 51). TL: Vietnam, Lamdong province, Fyan.

Vietnamese records: Sapromyza (Sapromyza) ctenophora; Sasakawa (2001, p. 46, discussion, distribution, in Vietnamese key, male genitalic figure); Sasakawa (2008, p. 44, in Oriental key, distribution).

Distribution: Vietnam.

23. Sapromyza (Sapromyza) septemnotata Sasakawa (2001, p. 53). TL: Vietnam, Laocai province, Sapa.

Vietnamese records: Sapromyza (Sapromyza) septemnotata; Sasakawa (2001, p. 46, discussion, distribution, in Vietnamese key, male genitalic figure, remarks); Sasakawa (2008, p. 45, in Oriental key, distribution); Shi et al. (2012, p. 186, diagnosis, distribution, habitus photo, in Chinese key, new to China).

Distribution: China (Yunnan, Hainan), Vietnam.

24. Sapromyza (Sapromyza) sexmaculata Sasakawa (2001, p. 52). TL: Vietnam, Vinhphuc province, Tamdao.

Vietnamese records: Sapromyza (Sapromyza) sexmaculata; Sasakawa (2001, p. 46, diagnosis, distribution, in Vietnamese key, male genitalic figure); Sasakawa (2003, p. 63, distribution, new to Laos); Sasakawa (2008, p. 45, in Oriental key, distribution); Shi et al. (2012, p. 186, diagnosis, distribution, habitus photo, in Chinese key, new to China).

Distribution: China (Zhejiang, Hainan), Laos, Vietnam. 
25. Sapromyza (Xenosapromyza) cinctipes (Meijere, 1910, p.125 (Lauxania (Sapromyza))). TL:Indonesia, Java, Depok.

Vietnamese records: Sapromyza (Xenosapromyza) cinctipes; Sasakawa (2001, p. 46, distribution, in Vietnamese key, new to Vietnam).

Distribution: Indonesia, Vietnam.

Genus Sciasmomyia Hendel, 1907

TS: Sciasmomyia meijerei Hendel, 1907.

26. Sciasmomyia meijerei Hendel (1907, p. 234). TL: Vietnam, Tonkin.

Vietnamese records: Sciasmomyia meijerei; Hendel (1907, p. 234, description); Hendel (1908, p. 18, list, figure of head and wing); Shewell (1977, p. 193, in Oriental Catalog); Sasakawa (2001, p. 39, previously known species in Vietnam); Shi, Gaimari, and Yang (2013, p. 420, description, diagnosis, habitus photo, key to species, male and female genitalic figure, new to China, remarks); Sciasmomyia demeijeri; Hendel (1925, p. 107, Misspelling, in key).

Distribution: China (Fujian, Jiangxi, Zhejiang), Vietnam.

27. Sciasmomyia thaii Shi et al. (2013, p. 428). TL: Vietnam, Vinhphuc province, Tamdao National park.

Vietnamese records: Sciasmomyia thaii; Shi et al. (2013, p. 428, description, diagnosis, distribution, habitus photo, key to species, male and female genitalic figure, remarks).

Distribution: Vietnam.

Genus Steganopsis Meijere, 1910

TS: Steganopsis pupicola Meijere, 1910.

28. Steganopsis japonica Sasakawa (1998, p. 68). TL: Japan, Ryukyu Islands, Kainan, Ishigaki Island.

Vietnamese records: Steganopsis japonica; Shatalkin (2000, p. 38, in Palaearctic key, distribution); Schacht, Kurina, Merz and Gaimari (2004, p. 58, English translation of Shatalkin, 2000, p. 38); Lee et al. (2016, p. 47, description, distribution, habitus photo).

Distribution: Japan, Vietnam. 
29. Steganopsis pupicola Meijere (1910, p. 146). TL: Indonesia, Java, Jakarta.

Vietnamese records: Steganopsis pupicola; Sasakawa (2001, p. 39, distribution, new to Vietnam).

Distribution: Indonesia, Sri Lanka, Vietnam.

\subsection{Tribe Trigonometopini Becker, 1905}

According to Papp (2007), the tribe Trigonometopini consists of 10 genera in the world. In Vietnam, following 4 genera are recorded: Luzonomyza, Protrigonometopus, Shatalkinella and Tetroxyrhina.

\section{Genus Luzonomyza Malloch, 1929}

TS: Trigonometopus bakeri Bezzi, 1913.

30. Luzonomyza sasakawai Papp (2007, p. 137). TL: Vietnam, Cucphuong, Ninhbinh province.

Vietnamese records: Luzonomyza sasakawai; Papp (2007, p. 137, description, head photo, key to species, remarks).

Distribution: Thailand, Vietnam.

31. Luzonomyza vietnamensis Papp (2007, p. 139). TL: Vietnam, Hatinh province, Huongson.

Vietnamese records: Luzonomyza vietnamensis; Papp (2007, p.139, description, head photo, key to species, remarks).

Distribution: Vietnam.

\section{Genus Protrigonometopus Hendel, 1938}

TS: Protrigonometopus maculifrons Hendel, 1938.

32. Protrigonometopus maculifrons Hendel (1938, p. 3). TL: China, Jiangsu.

Vietnamese records: Protrigonometopus maculifrons; Sasakawa (2001, p. 43, distribution, new to Vietnam).

Distribution: China (Jiangsu), Japan, Korea, Vietnam.

33. Protrigonometopus ornatus Papp (2007, p. 146). TL: Vietnam, Sin Chai. 
Vietnamese records: Protrigonometopus ornatus; Papp (2007, p. 146, key to species, male genitalic figure).

Distribution: Vietnam.

\section{Genus Shatalkinella Papp, 2007}

TS: Shatalkinella marginata Papp, 2007.

34. Shatalkinella deceptor (Malloch, 1927b, p. 167 (Sapromyza)). TL: Taiwan, Hokutu.

Vietnamese records: Protrigonometopus deceptor; Shatalkin (1997, p. 167, diagnosis, new to Vietnam); Shatalkinella deceptor; Papp (2007, p.1 58, key to species).

Distribution: China (Taiwan), Vietnam.

\section{Genus Tetroxyrhina Hendel, 1938}

TS: Trigonometopus submaculipennis Malloch, 1927.

35. Tetroxyrhina brunneicosta (Malloch, 1927b, p. 165 (Trigonometopus)). TL: Taiwan, Taitung County, Dawu Township (as Formosa: Paiwan District, Paroe).

Vietnamese records: Tetroxyrhina brunneicosta; Papp (2007, p. 160, new combination, examined material information); Shi, Gaimari and Yang (2017, p. 248, distribution, key to species, habitus and wing photo of holotype).

Distribution: China (Taiwan), Japan (Ryukyu Islands), Malaysia, Vietnam.

36. Tetroxyrhina cuneata (Shatalkin, 1997, p.165 (Trigonometopus cuneatus)). TL: Vietnam, Vinhphuc province, Tamdao.

Vietnamese records: Luzonomyza (Tetroxyrhina) cuneatus; Shatalkin (2000, p. 39, in Palaearctic key, distribution); Schacht et al. (2004, p. 59, English translation of Shatalkin, 2000, p. 39); Trigonometopus (Tetroxyrhina) cuneatus; Shatalkin (1997, p. 165, diagnosis); Tetroxyrhina cuneata; Shi et al. (2017, p. 248, distribution, key to species, habitus photo of holotype).

Distribution: China, Vietnam.

37. Tetroxyrhina sauteri (Hendel, 1912, p. 19 (Trigonometopus)). TL: Taiwan, Nantou County, Chi-Chi (as Formosa: Chip-Chip).

Vietnamese records: Trigonometopus (Tetroxyrhina) alboapicalis; Shatalkin (1997, p. 164, key to species, diagnosis); Tetroxyrhina sauteri; Shi et al. (2017, p. 248, 
key to species, diagnosis, redescription, male genitalic figure, distribution, habitus and wing photo of holotype).

Distribution: China (Yunnan, Zhejiang, Hainan, Taiwan), Thailand, Vietnam.

38. Tetroxyrhina submaculipennis (Malloch, 1927b, p. 164 (Trigonometopus)). TL: Taiwan, Taipei (as Formosa: Taihoku).

Vietnamese records: Trigonometopus (Tetroxyrhina) submaculipennis; Shatalkin (1997, p. 164, key to species, distribution); Sasakawa (2003, p. 61, distribution, new to Laos); Luzonomyza (Tetroxyrhina) submaculipennis; Shatalkin (2000, p. 39, in Palaearctic key, distribution); Schacht et al. (2004, p. 59, English translation of Shatalkin, 2000, p. 39); Tetroxyrhina submaculipennis; Shi et al. (2017, p. 248, distribution, key to species, habitus and wing photo of holotype).

Distribution: China (Gansu, Taiwan), Laos, Nepal, Vietnam.

39. Tetroxyrhina tinctipennis (Meijere, 1924b, p. 211 (Trigonometopus)). TL: Indonesia, Java.

Vietnamese records: Tetroxyrhina tinctipennis; Shi et al. (2017, p. 248, distribution, key to species, habitus photo of syntype); Trigonometopus (Tetroxyrhina) tinctipennis; Sasakawa (2001, p. 39, previously known species in Vietnam).

Distribution: Indonesia (Java), Malaysia, Vietnam.

\section{Genus Xangelina Walker, 1856}

TS: Xangelina basigutta Walker, 1856.

40. Xangelina basigutta Walker (1856b, p. 32). TL: Malaysia, Malacca.

Vietnamese records: Xangelina basigutta; Lee et al. (2016, p.48, description, distribution, habitus photo, new to Vietnam).

Distribution: Laos, Malaysia, Vietnam.

41. Xangelina heteroloba Sasakawa (2003, p. 60). TL: Laos, Vientiane province, Banvaneue, Phoukou Khouei.

Vietnamese records: Xangelina heteroloba; Lee et al. (2016, p. 49, description, distribution, habitus photo, new to Vietnam).

Distribution: Laos, Vietnam. 


\section{ACKNOWLEDGMENTS}

This work was supported by a grant from the National Institute of Biological Resources (NIBR), funded by the Ministry of Environment (MOE) of the Republic of Korea (NIBR201704201). I am grateful to the members of the Faculty of Biology, Dalat University for providing an intellectually inspiring environment for this study.

\section{REFERENCES}

Becker, T. (1905). Cyclorrhapha Schizometopa: Holometopa. In Becker, T., Bezzi, M., Mertesz, K. \& Stein, P. (Eds): Katalog der palaarktischen Dipteren ( $^{\text {th }}$ ed., pp. 1-273). Budapest, Hungary: Wesselényi.

Bezzi, M. (1913). Studies in Philippine Diptera. The Philippine Journal Science, 8, 305332.

Fallén, C. F. (1810). Specim. entomolog. novam Diptera disponendi methodum exhibens. Lundae, Sweden: Litteris Berlingianis Publishing.

Fallén, C. F. (1820). Ortalides Sveciae. Lundae, Sweden: Litteris Berlingianis Publishing.

Fallén, C. F. (1826). Supplementum Dipterorum Sveciae. Londini Gothorum, Sweden: ex officinal Berlingiana.

Hendel, F. (1907). Neue und interessante Dipteren aus dem kaiserl. Museum in Wien. (Ein Beitrag zur Kenntnis der acalyptraten Musciden.). Wiener Entomologische Zeitung, 26, 223-245.

Hendel, F. (1908). Diptera. Fam. Muscaridae. Subfam. Lauxaninae. Wytsman's Genera Insectorum fasc, 68, 1-66.

Hendel, F. (1912). Neue Muscidae Acalypteratae. Wiener Entomologische Zeitung, 31, 1-20.

Hendel, F. (1925). Neue ubersicht die Bisher Bekannt Gewordenen Gattungen der Lauxaniiden, nebst Beschreibung neuer Gattungen u. Arten. Encyclopedie Entomologique Ser B II Dipt, 2, 102-112.

Hendel, F. (1938). Muscaria holometopa (Dipt.) aus China im Naturhistorischen Reichstnuseum zu Stockholm. Arkiv for Zoologi Stockholm, 30(3), 1-13.

Kertész, K. (1913). H. Sauter's Formosa-Ausbeute. Lauxaniinae. (Dipt.). Ann. Hist.-Nat. Mus. Natl. Hung, 11, 88-102.

Kertész, K. (1915). H. Sauter's Formosa-Ausbeute. Lauxaniinae. II. Annls hist.-nat. Mus. Natn. Hung., 13, 491-534.

Lee, H. S., Bae, Y. S., Ram, K. D., Lee, J. W., Choi, J. K., Kim, Y. H., Choi, D. S., Byun, H. W., Jeon, M. J., \& Nguyen, V. K. (2016). Biodiversity of Southern Vietnam - Insects. Incheon, Republic of Korea: National Institute of Biological Resources. 
Lee, H. S. (2016). A checklist of the family Lauxaniidae (Diptera, Lauxanioidea) in Vietnam. Dalat University Journal of Science, 6(4), 493-515.

Linnaeus, C. (1758). Systema Naturae per regna tria naturae, secundum classes, ordines, genera, species, cum characteribus, differentiis, synonymis, locis. Holmiae, Sweden: Impensis Direct. Laurentii Salvii.

Macquart, P. J. M. (1835). Histoire Naturelle des insects. Dipteres. Paris, France: Librairie Encyclopédique de Roret.

Macquart, P. J. M. (1848). Dipteres exotiques nouveaux ou peu connus. Suite du 2. Me supplement. Mem. Soc. R. Sci. Agric. Arts, Lille, 1847(2), 161-237.

Macquart, P. J. M. (1851). Dipteres exotiques nouveaux ou peu connus. Suite du 4e supplement publie dans les memoires de 1849. Mem. Soc. R. Sci. Agric. Lille, 1850, 134-294.

Malloch, J. R. (1927a). Fauna Sumatrensis (Beitrag Nr. 40). Sapromyzidae (Dipt.). Suppl. Ent. Ber., 15, 102-110.

Malloch, J. R. (1927b). H. Sauter's Formosa Collection: Sapromyzidae (Dipt.). Ent. Mitt., Ber., 16, 159-172.

Malloch, J. R. (1929). Notes on some Oriental sapromyzid flies (Diptera), with particular reference to the Philippines species. Proc. U.S. Natl. Mus., 74(2751), $1-97$.

Meijere, J. C. H. de (1910). Studien uber sudostasiatische Dipteren (4 ${ }^{\text {th }}$ ed.). Die neue Dipterenfauna von Krakatau. Tijdschr. Ent, 53, 58-194.

Meijere, J. C. H. de. (1914). Studien uber sudostasiatische Dipteren (9 $9^{\text {th }}$ ed.). Tijdschr. Ent. 57, 137-275.

Meijere, J. C. H. de. (1924a). Studien uber sudostasiatische Dipteren XV. Dritter Beitraz zur Kenntnis der sumatranischen Dipteren. Tijdschr. Ent. 67(Suppl.), 164.

Meijere, J. C. H. de (1924b). Studien uber sudostasiatische Dipteren XVI. Tijdschr. Ent. 67, 197-224.

Papp, L., Merz, B., \& Foldvari, M. (2006). Diptera of Thailand. A summary of the families and genera with references to the species representations. Acta Zoologica Academiae Scientiarum Hungaricae, 52(2), 97-269.

Papp, L. (2007). A review of the old world Trigonometopini Becker (Diptera: Lauxaniidae). Annales Historico-Naturales Musei Nationalis Hungarici, 99, 129-169.

Robineau-Desvoidy, J. B. (1830). Essai sur les myodaires. Paris, France: Imprimé par autorisation du Roi a I'Imprimerie Royal. 
Sasakawa, M. (1995). Lauxaniidae (Diptera) of Malaysia (Part 3). Insecta Matsumurana, $52,149-153$.

Sasakawa, M. (1998). Oriental Lauxaniidae (Diptera) (Part 1). Sci. Rep. Kyoto Pref. Univ., Hum. Env. \& Agr., 50, 49-74.

Sasakawa, M. (2001). Oriental Lauxaniidae (Diptera) (Part 2). Fauna of the Lauxaniidae of Viet Nam. Sci. Rep. Kyoto Pref. Univ., Hum. Env. \& Agr., 53, 39-94.

Sasakawa, M. (2003). Oriental Lauxaniidae (Diptera) (Part 4). Fauna of the Lauxaniidae of Laos. Sci. Rep. Kyoto Pref. Univ., Hum. Env. \& Agr., 55, 57-74.

Sasakawa, M. (2008). Oriental Lauxaniidae (Diptera) (Part 5). Fauna of the Philippines, with descriptions of two new genera and seven new species. Sci. Rep. Kyoto Pref. Univ., Life \& Env. Sci., 60, 39-59.

Sasakawa, M., \& Pong, T. Y. (1990). Lauxaniidae (Diptera) of Malaysia (Part 1). Esakia, 1, 123-136.

Schacht, W., Kurina, O., Merz, B., \& Gaimari, S. D. (2004). Zweiflugler aus Bayern XXII (Diptera: Lauxaniidae, Chamaemyiidae). Entomofauna Zeitschrift Fur Entomologie, 25, 41-80.

Shatalkin, A. I. (1996). New and little-known species of flies of Lauxaniidae and Strongylophthalmyiidae (Diptera). Russian Entomological Journal, 4(1-4), 145157.

Shatalkin, A. I. (1997). East-Asian species of Lauxaniidae (Diptera). Genera Trigonometopus Mcq., Protrigonometopus Hendel. Dipterological Research, 8(3), 163-168.

Shatalkin, A. I. (2000). Keys to the Palaearctic flies of the family Lauxaniidae (Diptera). Zoologicheskie Issledovania, 5, 1-102.

Shewell, G. E. (1977). Family Lauxaniidae. In Delfinado, M. D. \& Hardy, D. E. (Eds.), A Catalogue of the Diptera of the Oriental Region, ( $3^{\text {rd }}$ ed., pp. 182-214). Honolulu, USA: University Press of Hawaii.

Shi, L., \& Yang, D. (2009). Two new species of the genus Noonamyia from Hainan in China (Diptera, Lauxaniidae). Zootaxa, 2017, 34-40.

Shi, L., \& Yang, D. (2014). Five new species of Minettia (Minettiella) (Diptera, Lauxaniidae) from China. ZooKeys, 449, 81-103.

Shi, L., Gaimari, S. D., \& Yang, D. (2013). Revision of Sciasmomyia Hendel (Diptera: Lauxaniidae), with eight new species. Zootaxa, 3691(4), 401-435.

Shi, L., Gaimari, S. D., \& Yang, D. (2017). Five new species of the genus Tetroxyrhina Hendel from China (Diptera, Lauxaniidae). Zootaxa, 4247(3), 246-280.

Shi, L., Li, W. L., \& Yang, D. (2012). Note on seventeen species from China of the genus Sapromyza, with description of two new species (Diptera, Lauxaniidae), Acta Zootaxonomica Sinica, 37(1), 185-198. 
Shi, L., Wang, J. C., \& Yang, D. (2011). Six homonymous species of three genera Homoneura, Minettia, and Sapromyza (Diptera, Lauxaniidae). Acta Zootaxonomica Sinica, 36(1), 80-83.

Shi, L., Wu, Z. Y., \& Yang, D. (2009). The genus Pachycerina Macquart, 1835 from China (Diptera: Lauxaniidae). Annales Zoologici, 59(4), 503-509.

Shi, L., Yang, D., \& Gaimari, S. D. (2011). Four new species from China and Southeast Asia (Diptera, Lauxaniidae, Homoneurinae). Revue Suisse de Zoologie, 118(4), 667-693.

Stuckenberg, B. R. (1971). A review of the old world genera of Lauxaniidae (Diptera). Ann. Natal Mus., 20, 499-610.

Walker, F. (1856a). Catalogue of the dipterous insects collected in Singapore and Malacca by Mr. A.R. Wallace, with descriptions of new species. J. Proc. Linn. Soc. Lond., Zool. 1, 4-39.

Walker, F. (1856b). Insecta Britannica, Diptera. London, England: L. Reeve. 